\title{
A common low back pain hiding tuberculous sacro-iliitis .
}

\author{
saoussen miladi ${ }^{1}$, Ben ayed ben ayed ${ }^{1}$, ALIA FAZAA ${ }^{1}$, meriem sellami $^{1}$, kmar ouenniche $^{1}$, \\ Leila Souabni $^{1}$, Selma kassab ${ }^{1}$, Selma Chekili ${ }^{2}$, kaouther ben abdelghani ${ }^{1}$, and ahmad \\ laatar $^{1}$ \\ ${ }^{1}$ University Hospital Center Mongi Slim \\ ${ }^{2}$ University of Tunis El Manar
}

November 16, 2021

\begin{abstract}
The sacroiliac joint is rarely affected by tuberculosis. Only few cases have been reported. Consequently, the diagnosis is often delayed. This case report highlights the importance of continued awareness for early detection and treatment of a tuberculous sacroiliac joint infection.
\end{abstract}

\section{A common low back pain hiding tuberculous sacro-iliitis}

Key Clinical Message: An early diagnosis of tuberculous sacro-iliitis requires a high index of clinical suspicion.

Isolated tuberculosis of the sacrum is rarely reported in the literature [1]. It usually presents as vague back pain [1]. We report the case of a 61-year-old healthy female with a history of low back pain lasting for 2 years with no radicular irradiation. Her pain increased while efforts and decreased at rest with no night awakeness and no relief under paracetamol. She did not report constitutional symptoms. A plain radiograph of the pelvis showed widening of the left sacroiliac joint space with erosions of the subchondral bone (figure1). Magnetic resonance imaging confirmed the left sacro-iliitis and showed a collection in the left sacro-iliac joint and in the left iliopsoas muscle with an extension to the sacral spinal canal (figure 2 and 3). A fistula was born from an opening inside the sacral spinal canal, along the first sacral nerve root, to an outside opening in the left gluteal area, resulting in an abscess measuring $3.8 \times 14.4 \mathrm{~cm}$. Considering these aspects, the most likely diagnosis was tuberculous sacro-iliitis. Percutaneous drainage of the abscess was performed. Smears of the aspirated material did not reveal acid fast bacilli, the culture on Lowenstein-Jensen medium was negative but PCR performed on the specimen was positive for Mycobacterium tuberculosis.

Acknowledgements: None.

Conflict of interest: None.

Draft manuscript prepared by Ben Ayed H and Miladi S. Improvements to draft made by the other coauthors.

\section{References:}

[1] Prakash J. Sacroiliac tuberculosis - A neglected differential in refractory low back pain - Our series of 35 patients. J Clin Orthop Trauma. 2014; 5(3): 146-153. 


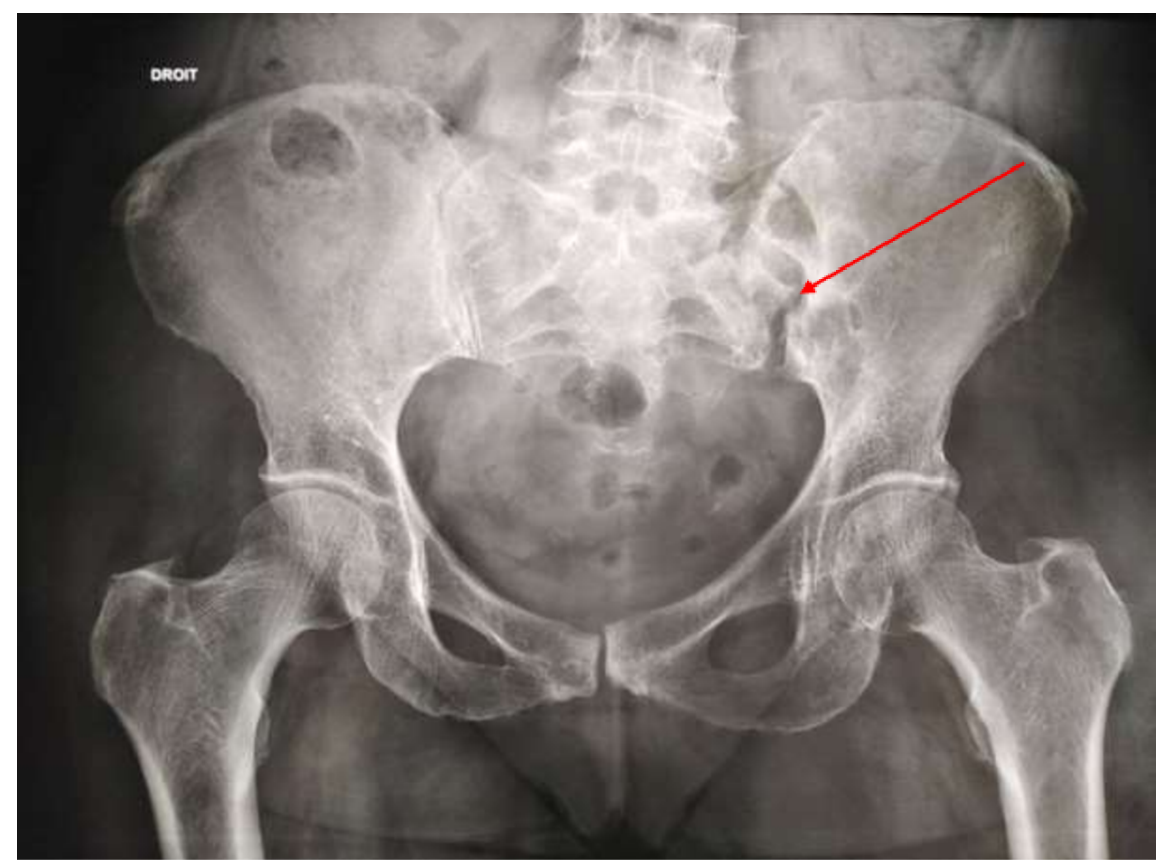

Figure 1: Plain radiograph of the pelvis showed widening of the left sacroiliac joint space with erosions of the subchondral bone

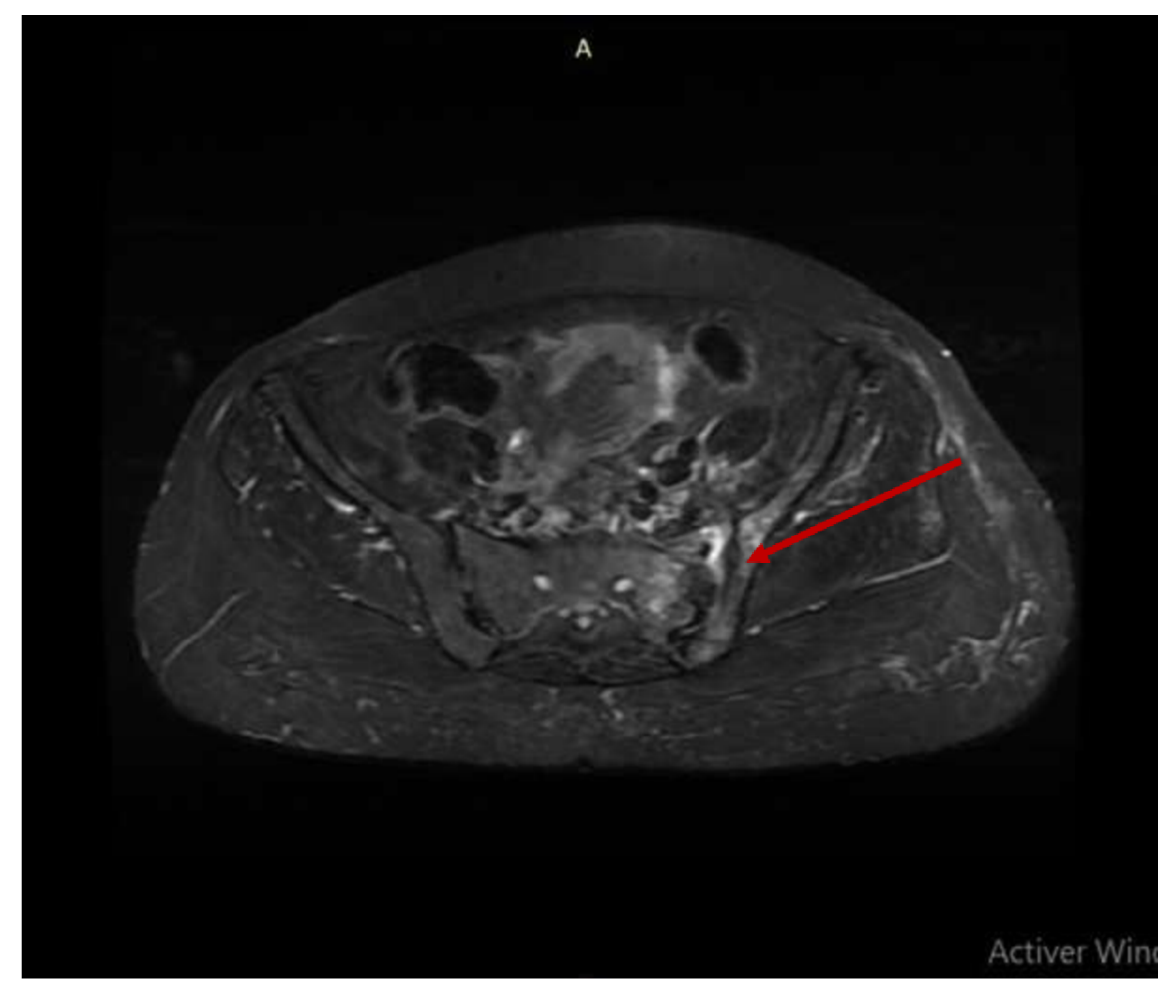

Figure 2: Magnetic resonance imaging (MRI) of the pelvis showing a left sacro-iliitis and a collection in the left sacro-iliac joint (arrow). 


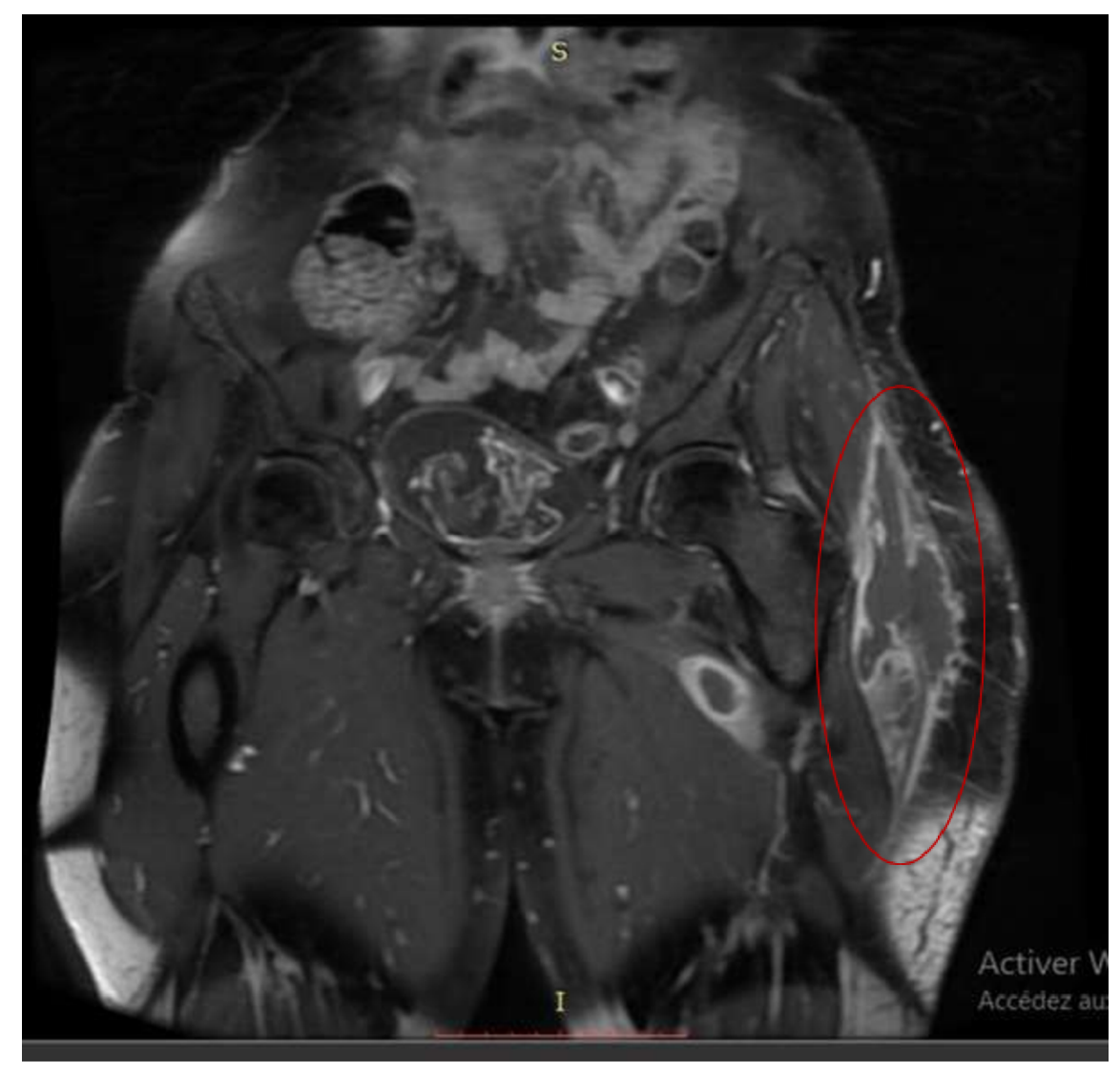

Figure 3: MRI image of pelvic and bilateral femur showing extension of the abscess distally around the left greater trochanter 